am-2019-13354z

Supplementary material

\title{
Dual-blockade immune checkpoint for breast cancer treatment based on a tumor-penetrating peptide assembling nanoparticle
}

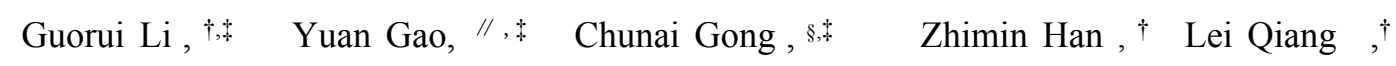
Zongguang Tai, ${ }^{\dagger}$ Jing Tian*, ${ }^{\dagger}$, Shen $\mathrm{Gao}^{*}, \uparrow$

†Department of Pharmacy, Changhai Hospital, Second Military Medical University, Shanghai 200433, China

" Department of Clinical Pharmacy and Pharmaceutical Management, Fudan University School of Pharmacy, Shanghai 201203, China

§Department of Pharmacy, Shanghai Ninth People's Hospital, Shanghai Jiao Tong University School of Medicine, Shanghai 200011, P. R. China

$\$$ Equally contributed to this work

* Corresponding authors

Tel./fax: +86 21 81873715, liullk@126.com (S. Gao)

Tel./fax: +86 2181873715 ,smmutj@126.com(J Tian) 
A

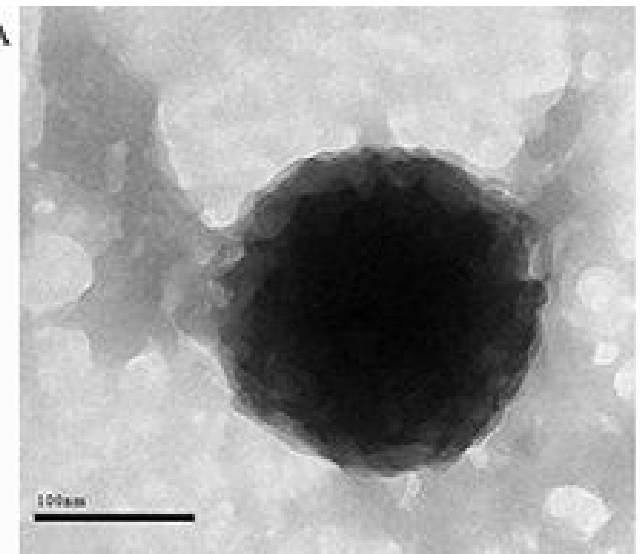

B

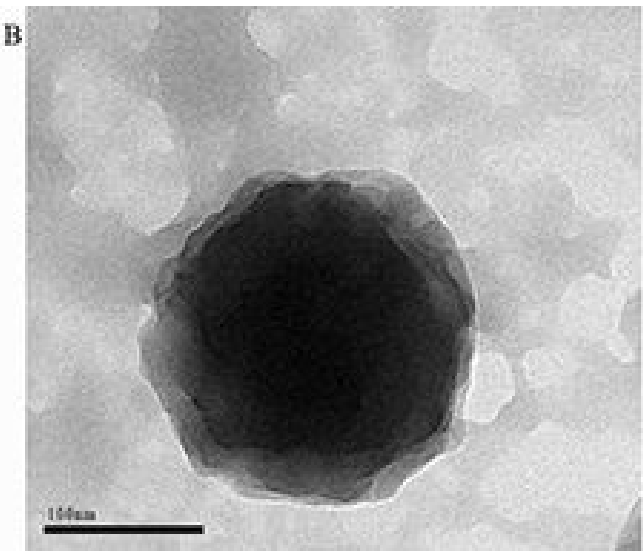

Figure S1. Transmission electron microscopy (TEM) images of 1MT-CHL (A) and Blank-CHL (B).

$A$

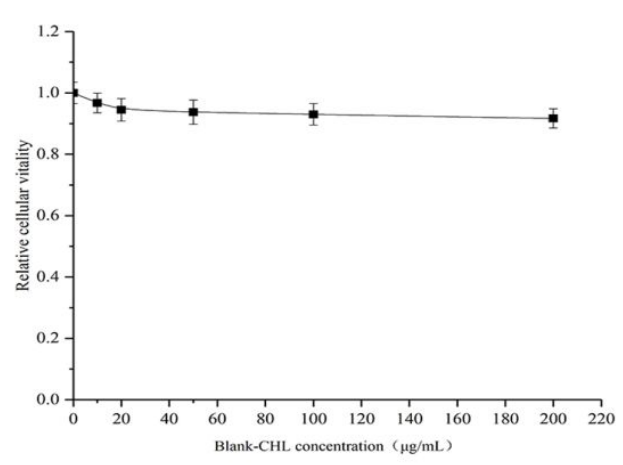

B

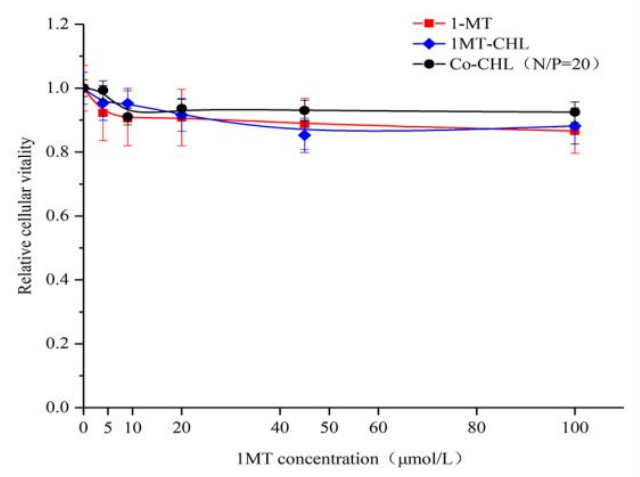

Figure S2. In vitro cytotoxicity against 4T1 cells. (A) In vitro cytotoxicity of blankCHL against 4T1 cells at different concentrations. (B) Cytotoxicity of free 1-MT, 1MTCHL, and Co-CHL (N/P $=20)$ to $4 \mathrm{~T} 1$ cells after $24 \mathrm{~h}$ of incubation. Data are expressed as the mean $\pm \operatorname{SD}(n=6)$. 

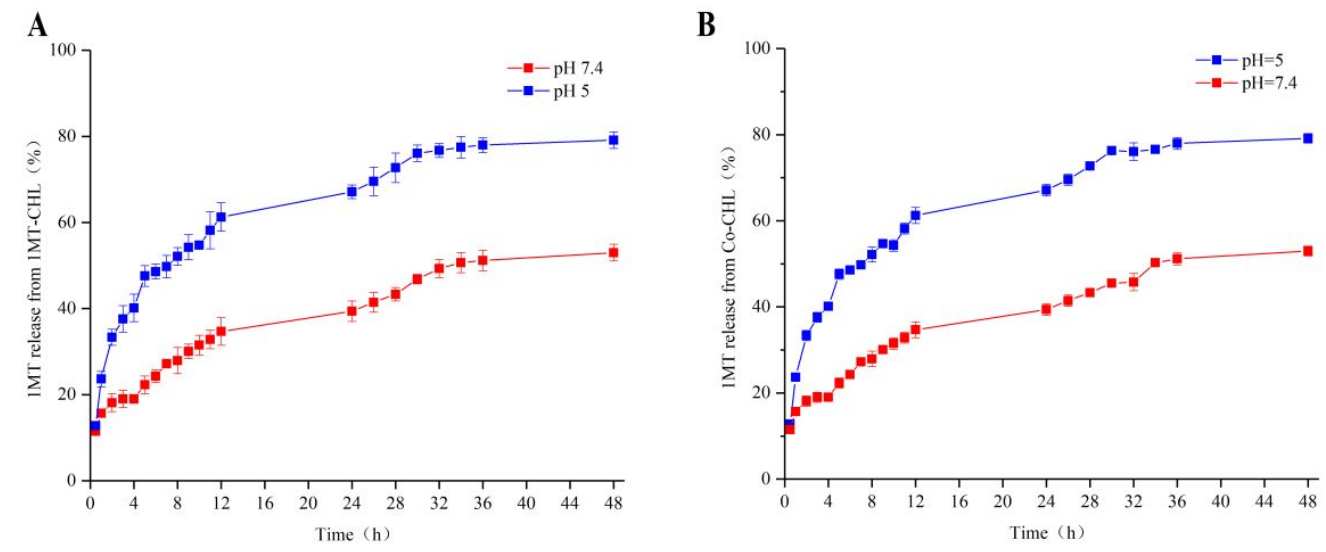

Figure S3. Profiles of 1-MT release from 1MT-CHL(A) and Co-CHL(B) at $\mathrm{pH} 5$ and 7.4. Data are expressed as the mean $\pm \mathrm{SD}(\mathrm{n}=3)$.
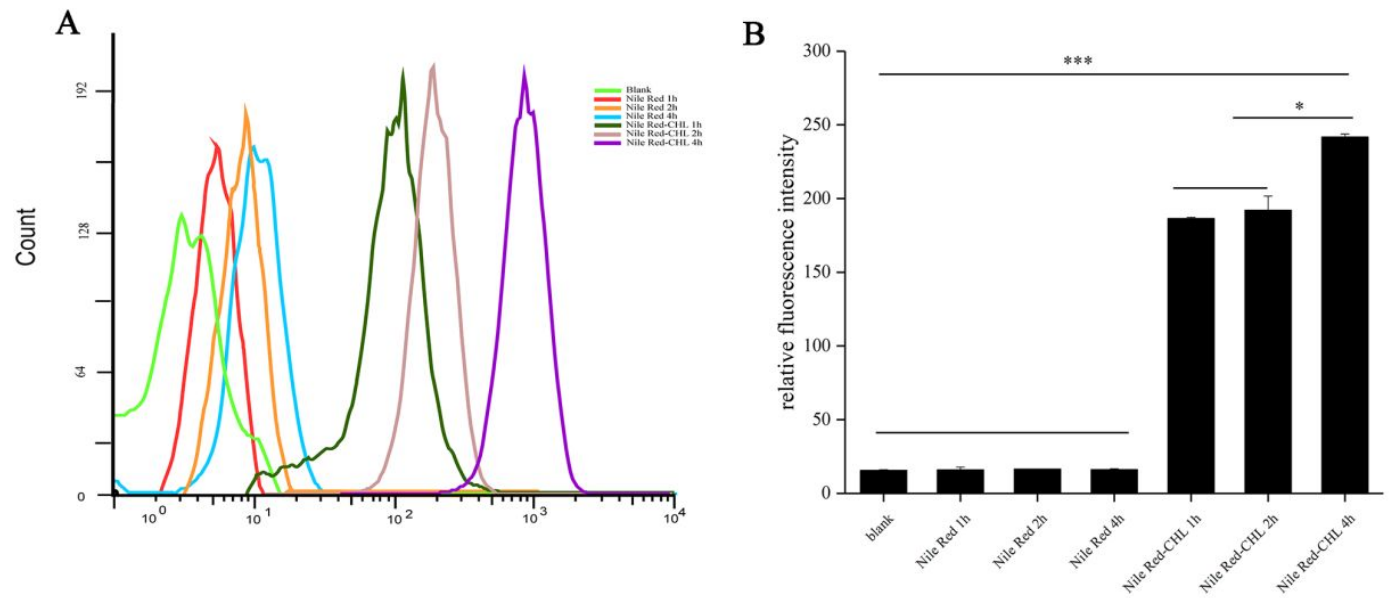

Figure S4. Cellular uptake of Nile Red. (A) Flow cytometry analysis of the amount of

Nile red internalized by $4 \mathrm{~T} 1$ cells after 1,2 , and $4 \mathrm{~h}$. (B) Quantification of relative fluorescence intensity between each treatment group and the blank group $(\mathrm{n}=3) .{ }^{*} p<$ $0.05, * * p<0.01, * * * p<0.001$. 
A

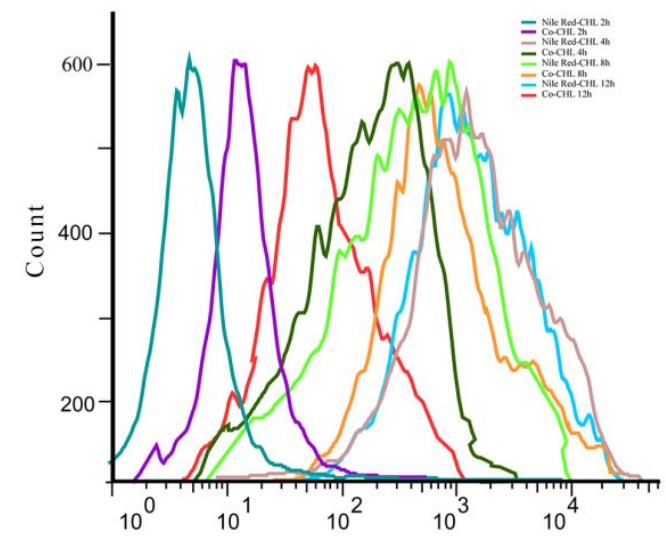

B

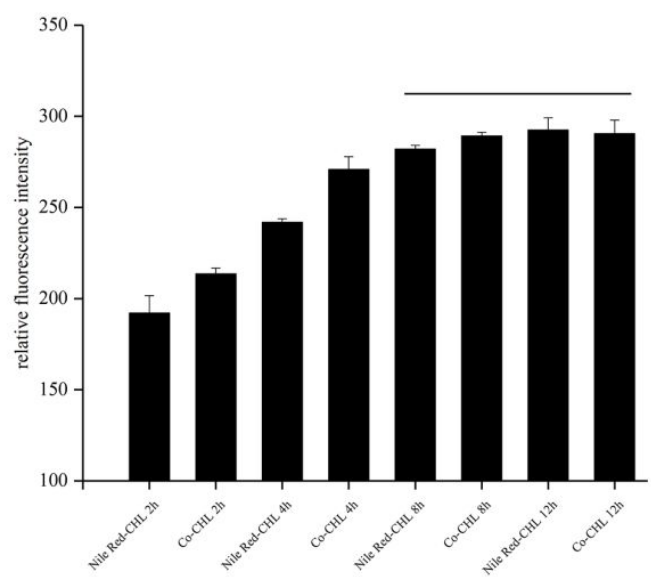

Figure S5. Cellular uptake of Nile Red-CHL and Co-CHL. (A) Flow cytometry analysis of the amount of Nile Red internalized by $4 \mathrm{~T} 1$ cells after 2, 4, 8, and $12 \mathrm{~h}$. (B) Quantification of relative fluorescence intensity between each treatment group and the blank group $(\mathrm{n}=3) . * p<0.05, *^{* *} p<0.01, * * * p<0.001$.

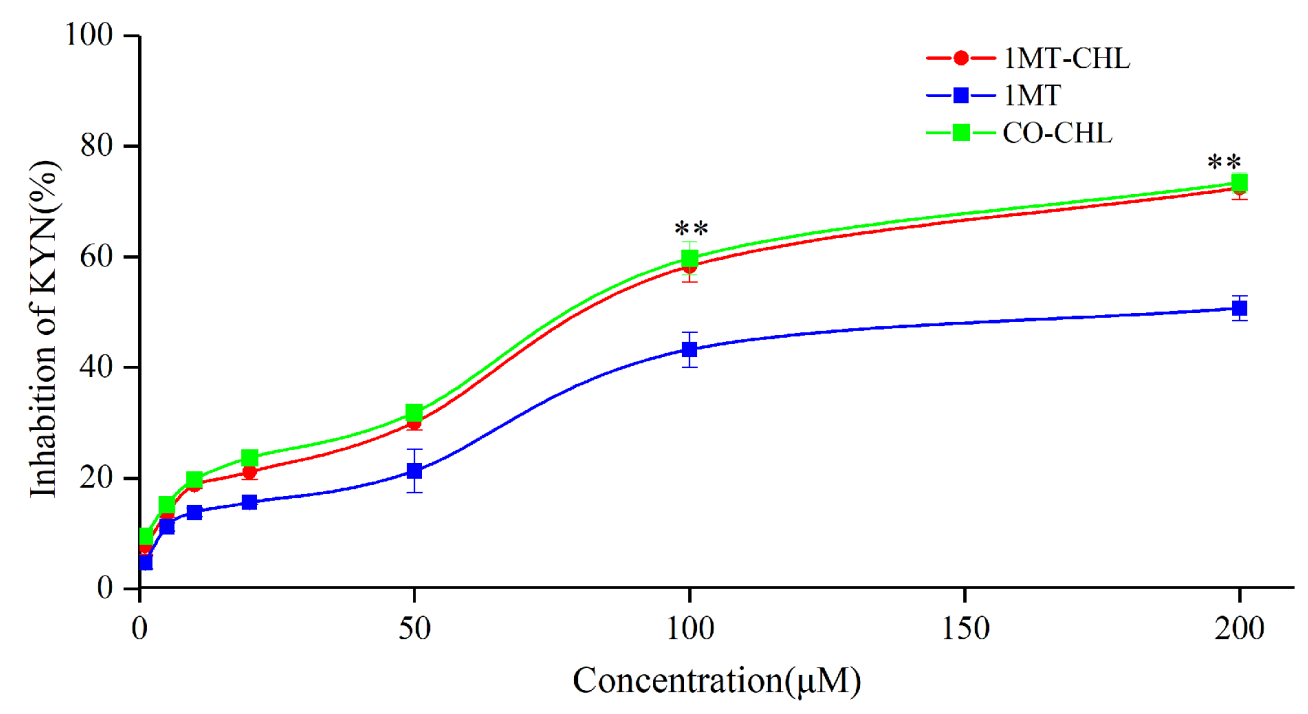

Figure S6. IDO enzymatic activity was measured as the inhibition of kynurenine production after free 1-MT ,1MT-CHL, and Co-CHL treatments. Data are expressed 
as the mean $\pm \operatorname{SD}(n=6)$. 